\title{
Design and Product Development of a Customized Lower Exoskeleton Rehabilitation mechanism
}

\author{
KuoChung Wang ${ }^{1, a}$, HuangKuang Kung ${ }^{2, b}$, ChinYu Wang ${ }^{2, c}$ \\ ${ }^{1}$ Phd Student, Department of Mechanical Engineering, Cheng Shiu University, Taiwan \\ ${ }^{2}$ Professor, Department of Mechanical Engineering, Cheng Shiu University, Taiwan \\ email: awe.lov@yahoo.com.tw, bhkkung@gclond.csu.edu.tw, ccywang333@yahoo.com.tw
}

\section{Keywords: Lower Exoskeletons; Rehabilitation mechanism; CAD/CAE}

\begin{abstract}
This study makes use of (1) LifeMod biomechanics software to construct customized user parameters; (2) the load conditions of hip joint and knee joint created by software analysis to serve as a basis of motor rating selection; (3) foot plantar pressure measurement plate and gesture recognition sensors to accomplish the monitoring and warning respectively of the rehabilitation motion. Based on the above-mentioned practice and procedure, the developed rehabilitation mechanism possesses the advantages of shortening design cycle, increasing added-value, improving product quality and enhancing product reliability. Above all, it is instrumental to the research and industry of rehabilitation products.
\end{abstract}

\section{Introduction}

This paper is to develop a customized lower extremity exoskeletons rehabilitation mechanism to assist post-operative patients to regain certain rehabilitation motions such as natural gait, squatting, etc. to achieve rehabilitation effectiveness.

Initially, the motions of pre-operative patients are recorded and analyzed by motion capture equipment to furnish customized mechanism with geometric design and provide the relative motion criteria of hip joint motor and knee joint motor with reference during rehabilitation phase. Next, through the design software, both the geometry design of mechanism system and the size design of machine elements can be accomplished. In succession, by using analysis software, the correct contact pairs among motions of machine elements can be established to fulfil the kinematic and dynamic simulations and analysis of the developed rehabilitation mechanism. Finally, the above-mentioned mechanism will be realized by integrating two harmonic drives, one Maxon motor and one GALIL multi-axis motion control card to complete the development of a single-leg, 2-axis rehabilitation mechanism.

In recent two decades, the rehabilitation mechanism for exoskeleton is extensively developed. The exoskeleton systems for enhancing human body performance were cooperatively developed by many academic and business personnel. America company Yobotics has developed a single-knee exoskeleton walking aids equipment-RoboKnee which can effectively perform torque compensation for knee joint by keeping the relaxation of thigh muscles and achieving low-resistance motion [1-3]. UC Berkley [4] also has developed a limb end exoskeleton ( BLEEX ) structure. At present, UC Berkley has successfully developed two generations of lower limbs walk aids robot. BLEEX comprises backpack frame, power unit, communication equipment, stainless mechanical limbs, control unit and hydraulic-power component. Yoshiyuki Sankai of Tsukuba University has led a team composed of scientists and engineers. They developed the world first Hybrid Assistive Leg (HAL) in his Cybernics laboratory. In succession, Cyberdyne Company is established and makes batch production. The HAL wearer can walk freely in $4 \mathrm{~km} / \mathrm{h}$ speed and basic moving skills such as taking stairs easily [5]. Besides, K. H. Low of Nanyang Technological University, Singapore researched and developed NTU lower limbs exoskeleton system on the basis of ZMP balance theory. The ZMP-based exoskeleton robot not only can walk synchronously with wearer, but also can walk alone like two-leg robots [6]. Different from other exoskeleton robots, 
balance theory is its distinguishing feature. The famous Israel engineer Amit Goffer of Argo Medical Technologies has hosted a research to develop a rehabilitation robot to help the waist down paralyzed patients standing up, walking and taking stairs. "ReWalk" uses a pair of sticks to maintain the balance of body [7].

Different from the inherited researches, this paper makes use of design, analysis, and simulation software to rapidly develop a customized rehabilitation mechanism. Also, by the aid of software and sensors, it helps patient comfortably and efficiently carry out rehabilitation motion. In addition to the originality of concrete results such as design, analysis, manufacturing, and product development, the paper employs 2D sensors to measure the stress on the ankle joint to provide the under-recovery patients with correct proportion between load of hip joint motor and knee joint motor with respect to rotation angle when performing different lower extremity motions, thus achieving timely and right assistance from motors.

The research architecture and procedure are shown in Fig. 1. The corresponding development software, hardware, and sensors are shown in Fig. 2.

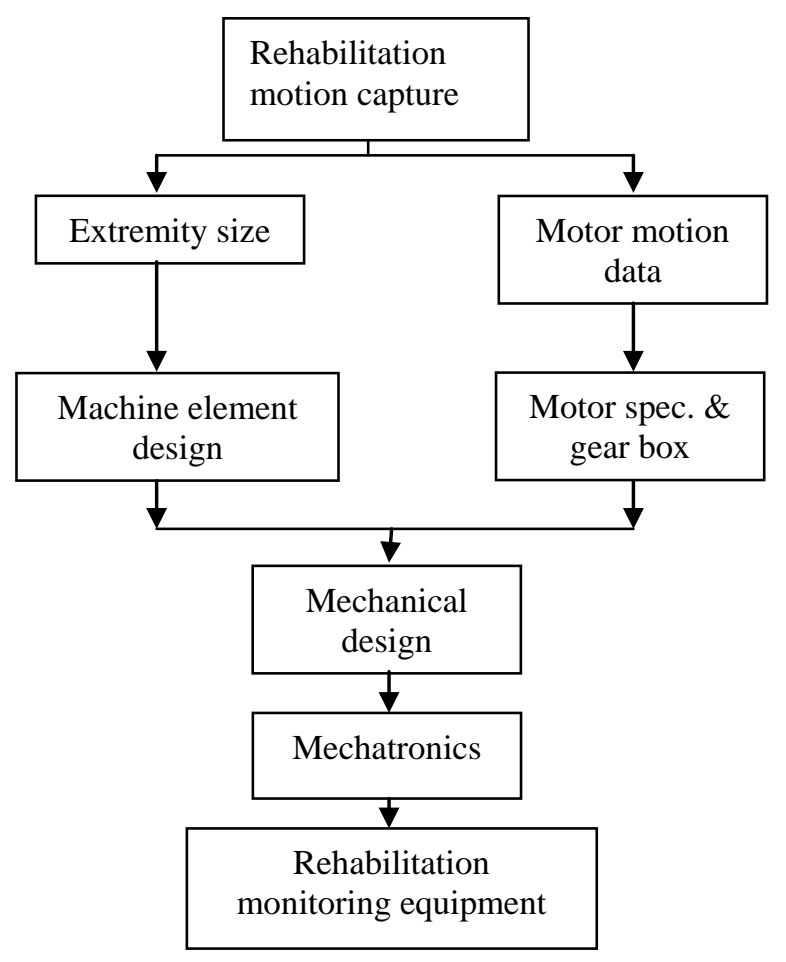

Fig. 1. Research architecture and procedure

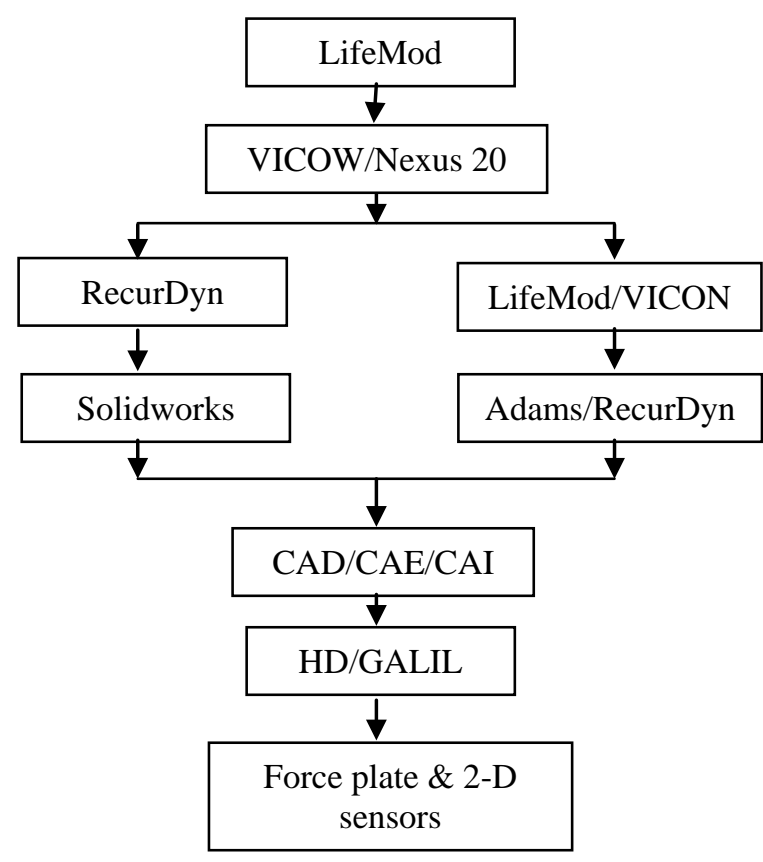

Fig. 2. The software/hardware for system

\section{Study methodologies}

Motion capture. The rehabilitation motions are recorded by the instrumentation of motion capture system NICON and 8 Bonita B10 camera along with software Nexus 2.2 ( with sampling rate $100 \mathrm{HZ}$ ) as shown in Fig. 3. The motion regularity of patient prior to operation is recorded to provide the two motors with the relation between motion and rotational angle, thus helping the patients accomplish rehabilitation easily and effectively. Those motion capture pre-set condition as shown Fig. 4. 

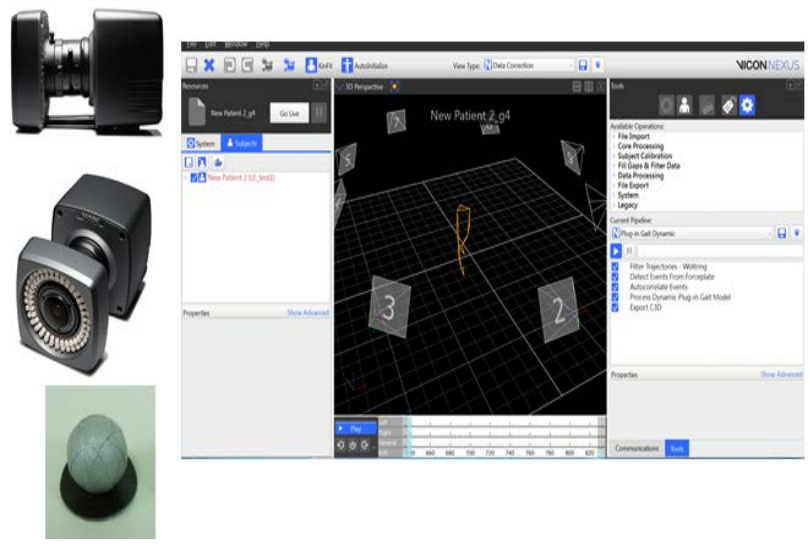

Fig. 3. The used motion capture system
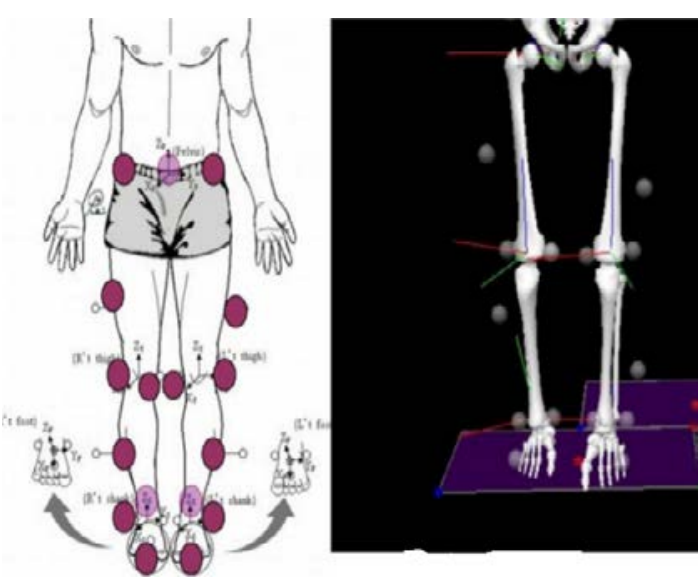

Fig.4. Pre-set condition based on human motion

Simulation results of biomechanics software. By utilizing biomechanics software LifeMod, the biomechanical data such as weight, bone length, muscle and ligament of patient are keyed in. After the SIL file captured in the rehabilitation motion is imported to LifeMod, the torque analysis of joints and foot plantar pressure can be performed. Based on the joint torque data, the specification of motor can be determined. In addition, the magnitude of reaction force on the foot plantar can be used to verify the measured data of foot pressure plate. Figure 5 illustrates ground reaction force condition, and the joint torques rules as shown Fig. 6.

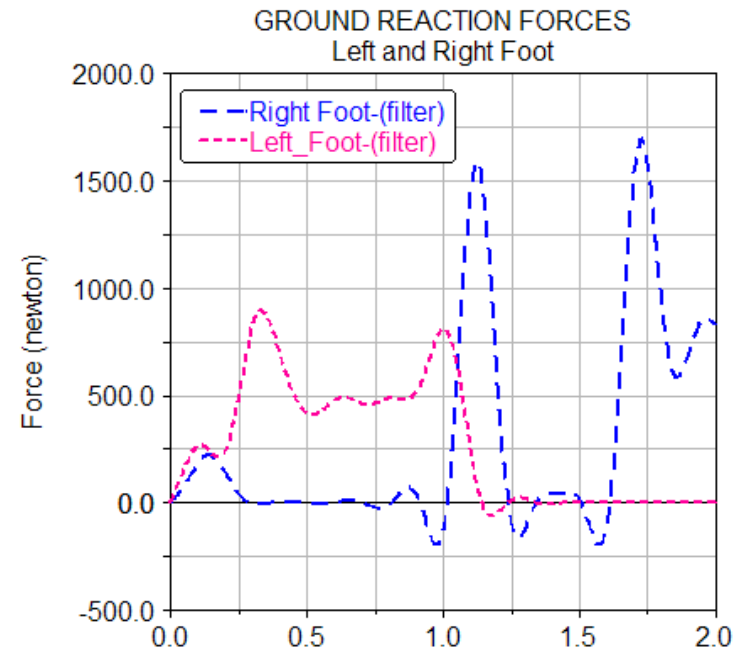

Fig. 5. Reaction force exerted on foot plantar

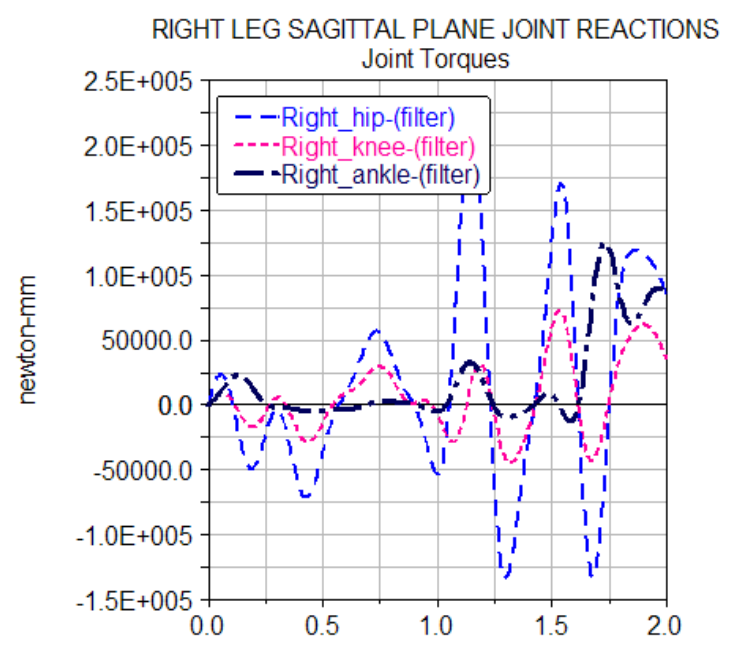

Fig. 6. The joint torque of the right lower

Rehabilitation mechanism design. According to the size of the lower extremity rehabilitation mechanism and the position constraints of motion, the machine of the rehabilitation mechanism and system design is carried out as shown in Fig. 7. 

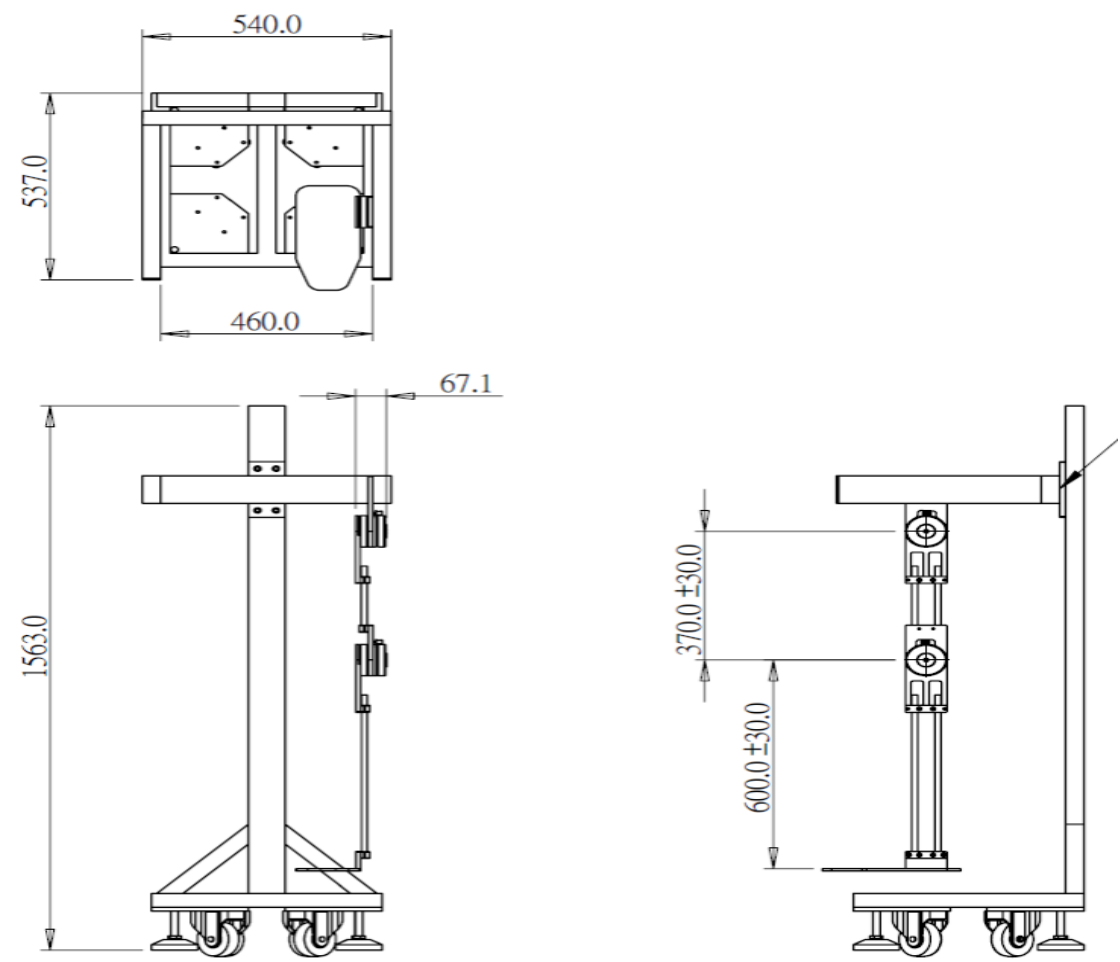

Fig. 7. The overall system design

Dynamic simulation of the proposed mechanism. According to the contact pair of the above-mentioned mechanism, the setting that meets the motion freedom is established. Based on the constraints or load condition of the proposed mechanism during rehabilitation, the motion analysis and analysing work of dynamics can be accomplished. Fig. 8 shows the mechanism constraints in using RecurDyn software. Fig. 9 demonstrates the load condition of mechanism. Next, Fig. 10 shows the motion analysis of the presented mechanism.

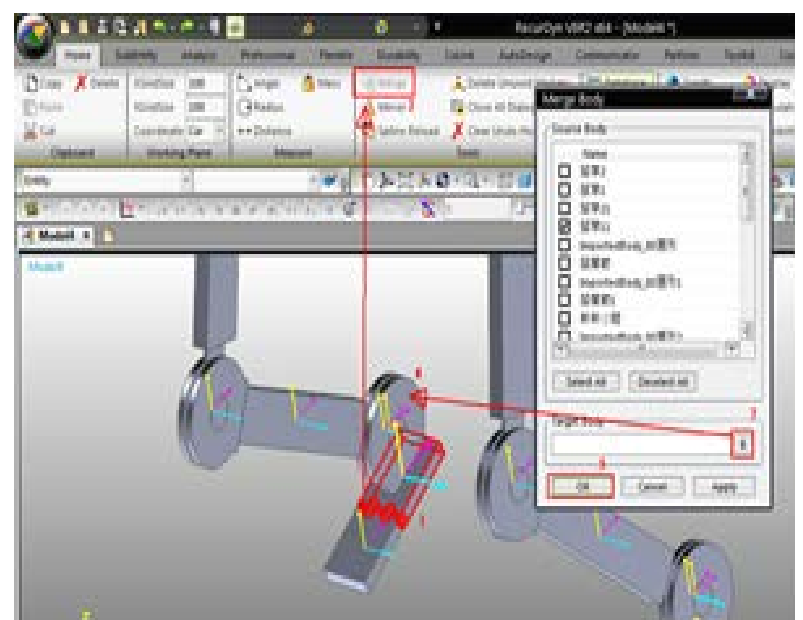

Fig. 8. Constraint condition setting

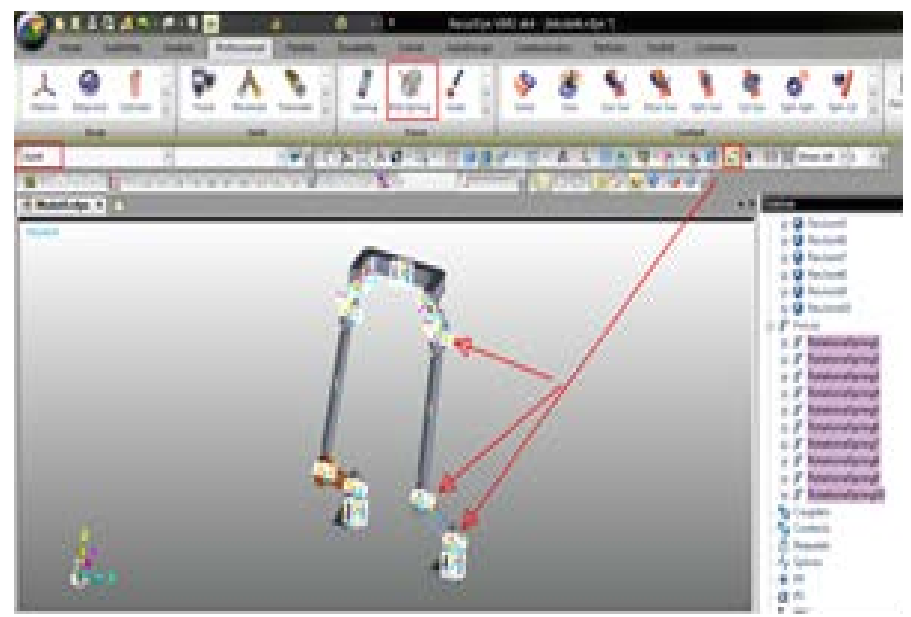

Fig. 9. Load condition setting 


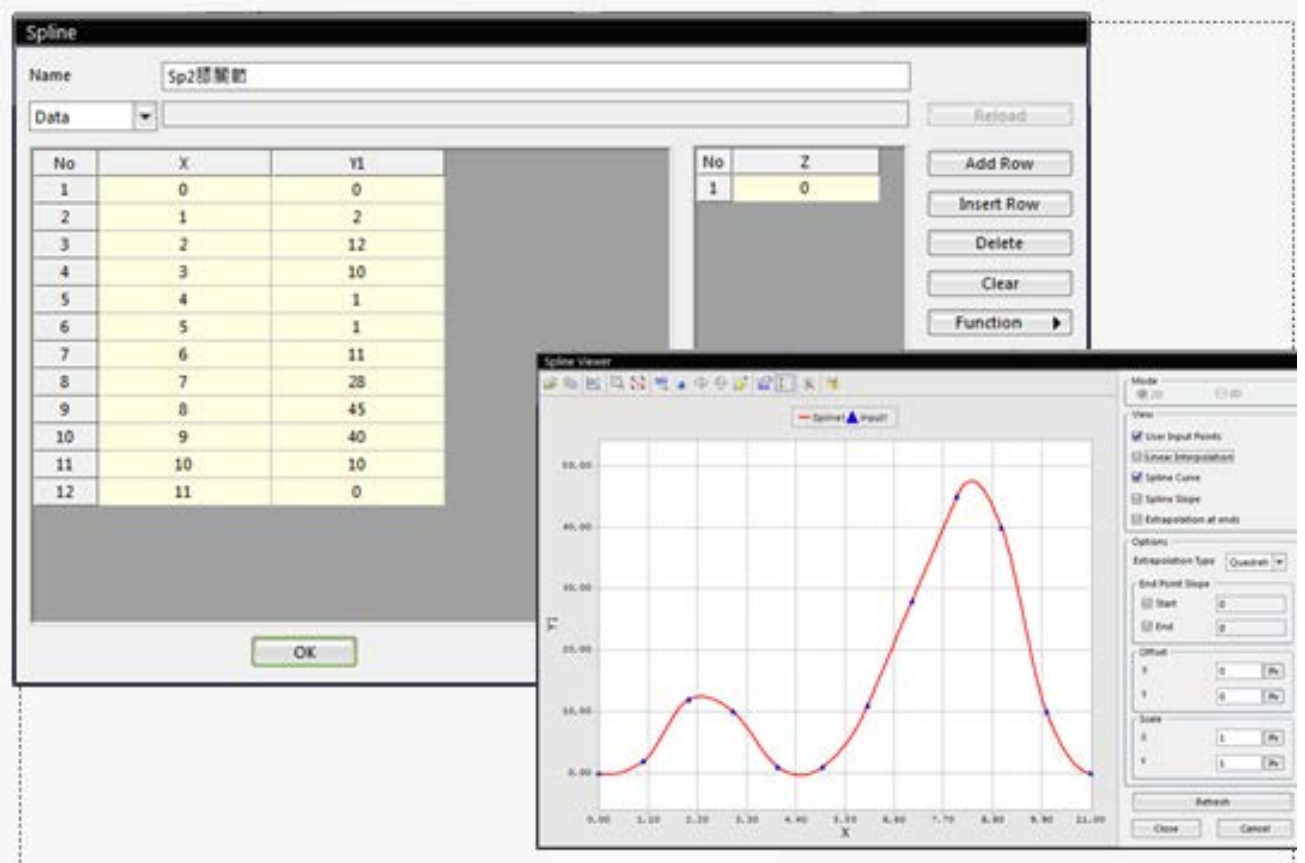

Fig. 10. Motion analysis of the lower extremity mechanism

\section{Rehabilitation mechanism and sensor system}

Outline of the intention-oriented vector. In this research, a 2-D force sensor is placed on the knee joint of patient to detect the signals of the self motion intention. Initially, the base vectors of hip joint and knee joint are defined. As shown in Fig. 11, the base vectors are tangent to the joint motion paths. To take one example, the 2-D sensor place in the ankle joint measures and achieves the magnitude of the intention vector $\vec{P}$ as shown in Fig. 12. With base vectors being $\vec{A}$ and $\vec{B}$, the vector $\vec{P}$ is given by

$$
\vec{P}=S \vec{A}+T \vec{B}
$$

The directions of motors are directed CCW of hip joint motion and CW of knee joint motion respectively. The angular speed and load torque yield the following relationship.

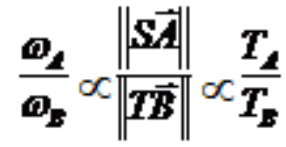

where $\omega_{A}$ and $\omega_{B}$ are angular velocites, and $T_{A}$ and $T_{B}$ are torques.

At present, the posture of lower extremity can be measured by motor encoder and the angle relationship shown in Fig. 12 can be measured by angle gauge. 


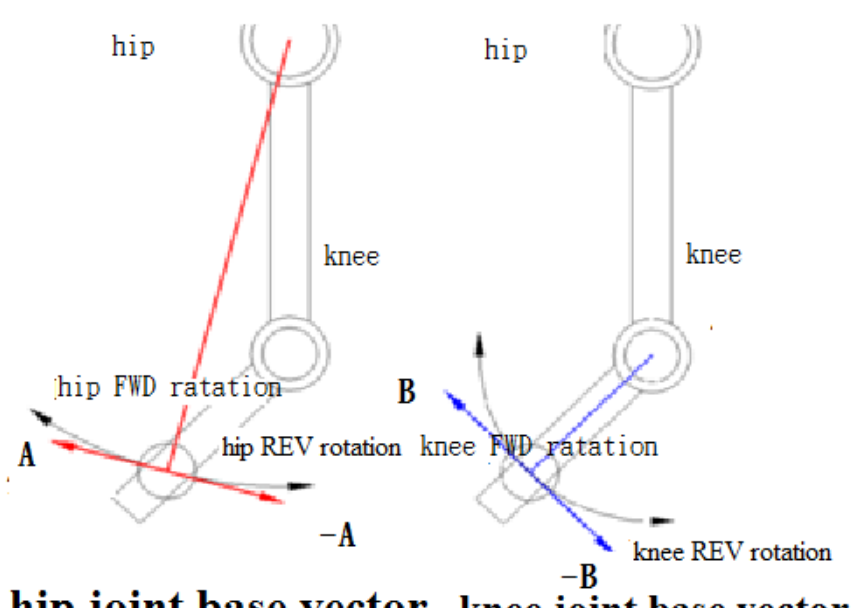

hip ioint base vector knee joint base vector

Fig. 11. Base vectors of hip and knee joint

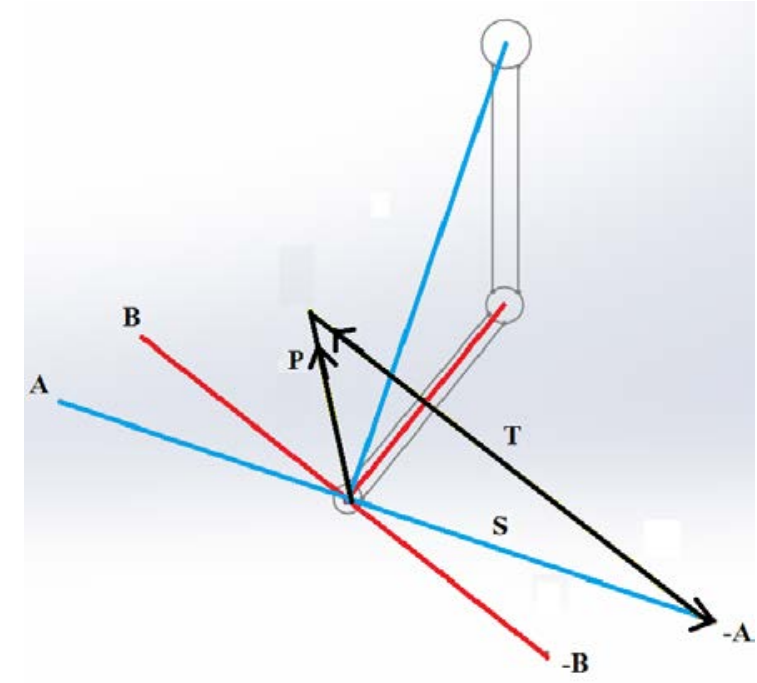

Fig. 12. The geometric relation between intention-oriented vector and motor

Sensor system of intention vector. For obtaining the motion relationship between the 2-D sensing of intention vector and motor, an experimental equipment is implemented with two drive motors being installed to validate the availability of the presented concept as shown in Fig. 13.

Foot plantar force plate. The foot plantar force plate can not only monitor the magnitude of the foot pressure and plantar force distribution, but also supervise the safety of user. More, the magnitude of foot plantar force can be employed as the boundary condition between software or used for varification. It therefore can furnish the result feasibility of software with quantitative condition. The dimension of force plate is $400 \mathrm{mmx} 600 \mathrm{~mm} x 8.25 \mathrm{~mm}$ which is a product of AMI with force capacity $\mathrm{Fx} / \mathrm{Fy} / \mathrm{Fz}$ being $500 / 500 / 100$ lbs. respectively and $1000 \mathrm{~Hz}$ sampling rate as illustrated in Fig. 14. Fig.15 shows the measurement results of a 62KG tester with fast walk gait.

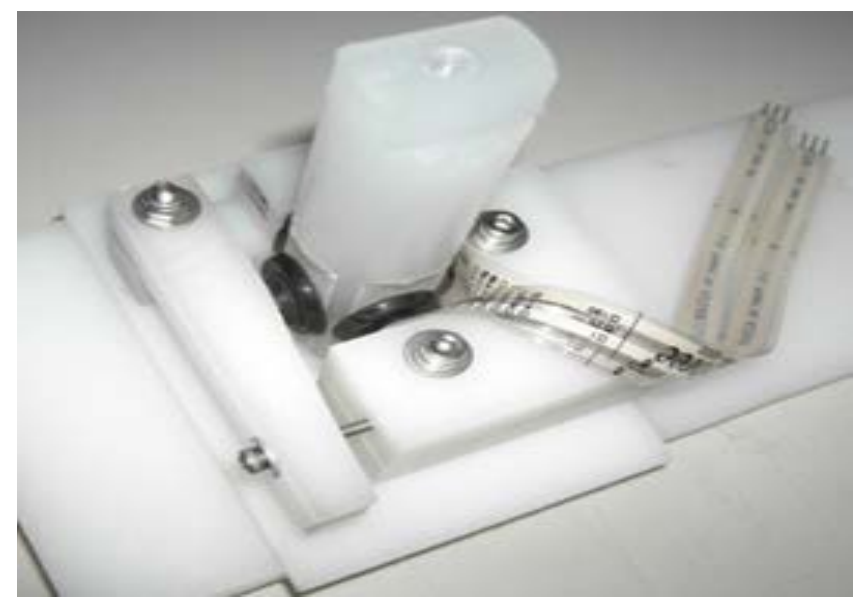

Fig. 13. 2-D sensor of intention vector

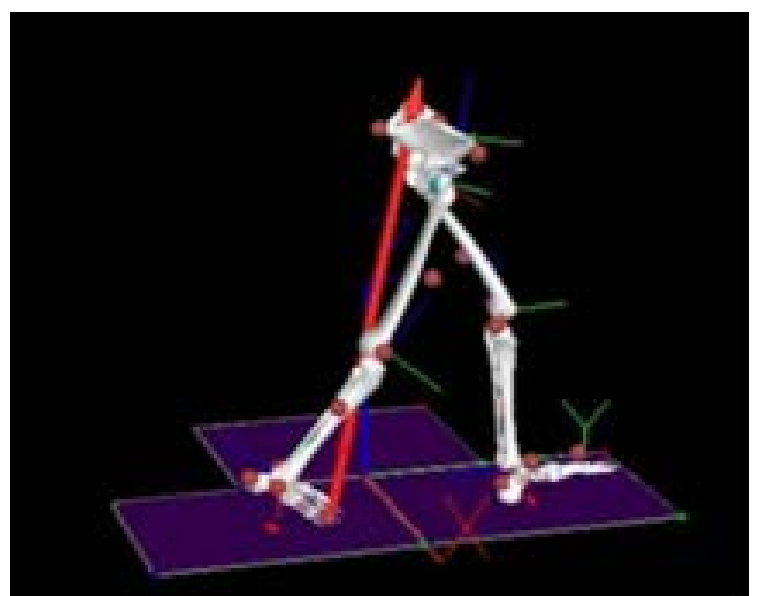

Fig. 14. The gait pattern of a fast walker tester 

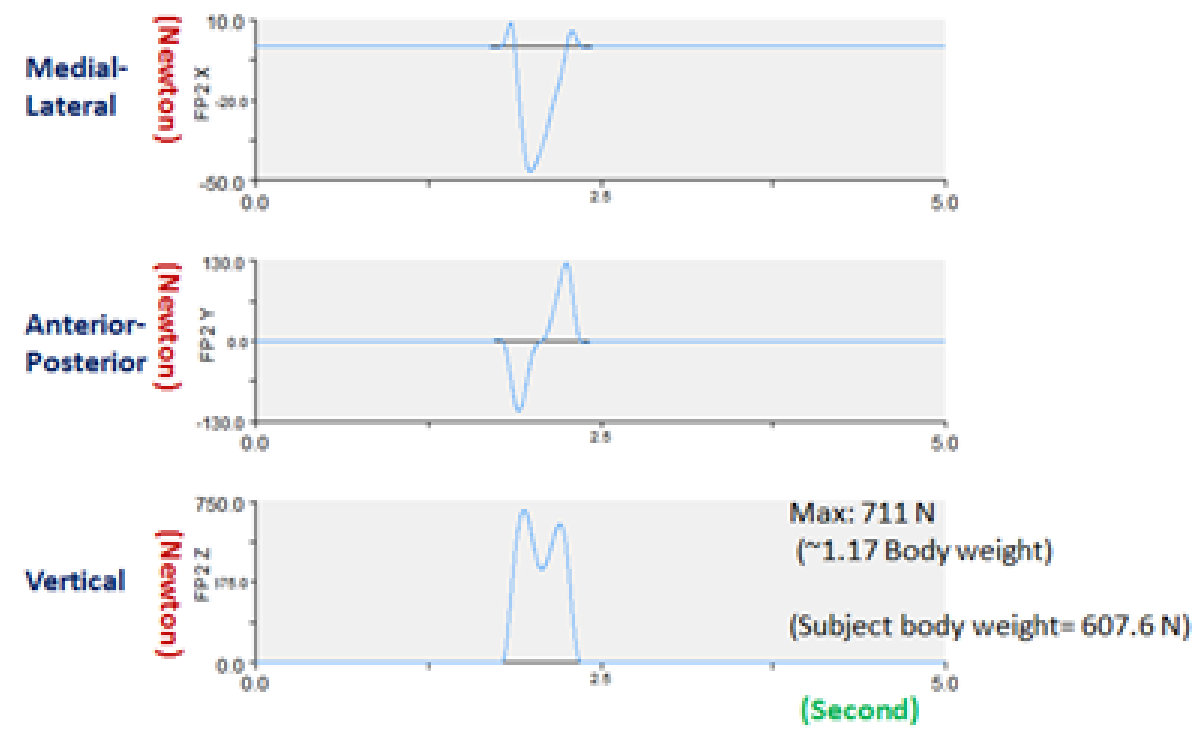

Fig. 15. The measurement results of fast walking

\section{Rehabilitation mechanism and mechatronics system}

Motor specification. According to the design condition, an exoskeleton rehabilitation mechanism of the left lower extremity is realized. The used brushless motor Maxon EC90(90 Watt) possesses the following parameters, as shown table 1.

Table 1 Parameters of motor

\begin{tabular}{cc|cc}
\hline Parameter & Value & Parameter & Value \\
\hline No-load speed & 2080rpm & Rated torque & 533mnm \\
Pre-set voltage & $40 \mathrm{~V}$ & Starting current & $21.1 \mathrm{~A}$ \\
Pre-set speed & $1610 \mathrm{rpm}$ & Rated current & $2.27 \mathrm{~A}$ \\
\hline
\end{tabular}

Encoder and Gear box. The RS422 product of MILE with 1600 pulses and two channels is used. The harmonic drive gear numbered SHD20-160 is used.

The driver. The Swiss-made TECHNOSOFI IDM240 intelligent servo driver system is employed.

The multi-axis controller. The 4-axis control card of GALIL company is utilized as shown in Fig. 16.

The motor and drive system. As shown in Fig. 16. The prototype rehabilitation mechanism is illustrated in Fig. 17.
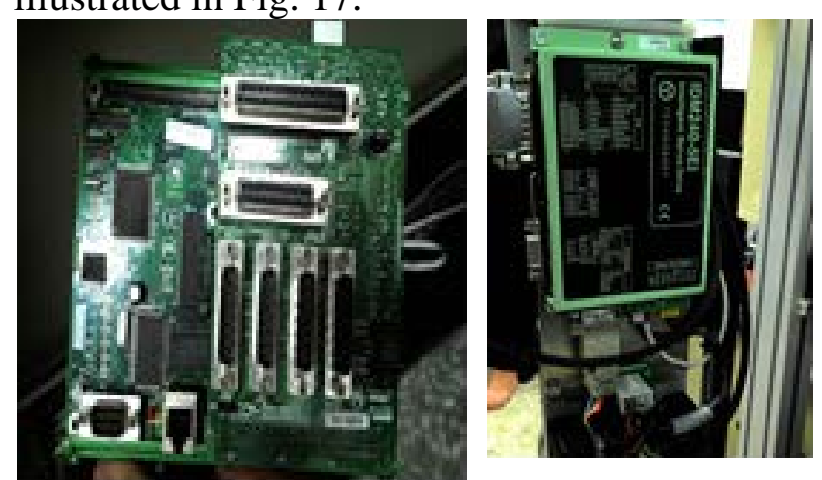

Fig. 16. Control card and drive system

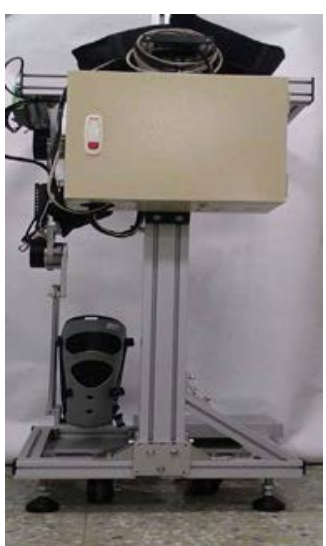

Fig.17. The rehabilitation mechanism product

\section{Summary}

This research presents a customized rehabilitation mechanism that is based on design, analysis, 
and simulation software. Each of software can provide predictable customized condition that determines the required hardware specification. Accordingly, the product quality can be promoted and the development period can be shortened. Therefore, the faster and more effective rehabilitation results for patients can be expected. Moreover, the ankle joint design based on intention vector can be equivalent to the rotation angles of electric motor for knee joint and hip joint by using the 2-D force sensor. The electric motor can therefore deliver timely and proper torque to assist the lower extremity rehabilitation. Finally, the proposed rehabilitation mechanism product is realized and implemented to validate the availability and reliability of the presented theory.

\section{Acknowledgements}

The authors would like to thank the financial support of National Science Council, Taiwan (Project: Engineering Analysis and Manufacturing of Human Knee Joints and its Prosthesis, NSC 99-2632-E-230-001-MY3).and NO:NSC99-2632-E-230-001-MY3. Special gratitude also belongs to Dr. Hsiao and Dr, Tsai of Biomechanics Laboratory I-Shou University who kindly supplied the precious motion capture and force plate measurement data to help the accomplishment of this research.

\section{References}

[1] B.J. Makinson, General Electric CO. Research and Development Prototype for Machine Augmentation of Human Strength and Endurance, Hardiman I Project, General Electric Report S-71-1056 [J], Schenectady, NY, 1971.

[2] Adam Zoss, H. Kazerooni, Andrew Chu. On the Mechanical Design of the Berkeley Lower Extremity Exoskeleton (BLEEX), IEEE/RSJ International Conference on Intelligent Robots and Systems, 2005: 3132-3139.

[3] Jerry E. Pratt, Benjamin T. Krupp, Christopher J. Morse, The RoboKnee. An Exoskeleton for Enhancing Strength and Endurance During Walking[C], Proceedings of the 2004IEEE International Conference on Robotics \& Automation, New Orleans, LA, April 2004: 2430-2435.

[4] Adam Zoos, H. Kazerooni, Andrew Chu. On the Mechanical Design of the Berkeley Lower Extremity Exoskeleton (BLEEX). IEEE Intelligent Robots and Systems Conference, August, 2005: 3465-3472.

[5] Hiroaki Kawamoto, Shigehiro Kanbe, Power Assist Method for HAL-3 Estimating Operator's Intention Based on Motion Information[C], Proceed125ing of the 2003IEEE International Workshop on Robots and Human Interactive Communication, Millbrae, California, USA, Oct. 31-Nov. 2, 2003: 67-72.

[6] Hiroaki KAWAMOTO, Yoshiyuki SANKAI. Power Assist Method Based on Phase Sequence Driven by Interaction between Human and Robot Suit[C], Proceedings of the 2004IEEE International Workshop on Robot and Human Interactive Communication, Kurashiki, Okayama Japan, Sep. 20-22, 2004: 491-496.

[7] Kama Moto H, Sankai Y. Comfortable power Assist control method for walking aid by HAL-5, IEEE Intel Conference on SMC.TP1B2, 2002: 880-912. 